\title{
ON CONJECTURES OF RIVIERE AND STRICHARTZ
}

\author{
BY CARLOS E. KENIG AND PETER A. TOMAS ${ }^{1}$
}

A. P. Calderón [1] showed that every bounded rational function of a real variable is a multiplier of $L^{p}, 1<p<\infty$. Littman, McCarthy and Rivière [5] showed this fails in $\mathbf{R}^{n}$ because $\left(\xi_{1}-\sum_{j=2}^{n} \xi_{j}^{2}+i\right)^{-1}$ is not a multiplier of $L^{p}\left(\mathbf{R}^{n}\right)$ if $1 \leqslant p<(2 n+2) /(n+2)$. Rivière conjectured that every bounded rational function is a multiplier of $L^{p}\left(\mathbf{R}^{n}\right)$ from some $p \neq 2$. We show this is false.

Theorem A. Let $\phi$ be in $L^{s} \cap L^{\infty}(\mathbf{R})$ for some $s, 0<s<\infty$. Then, $m\left(\xi_{1}, \xi_{2}\right)=\phi\left(\xi_{2}-\xi_{1}^{2}\right)$ is a multiplier of $L^{p}\left(\mathbf{R}^{2}\right)$ if and only if $p=2$.

R. Strichartz conjectured that there are no nontrivial Fourier multipliers of $L^{p}\left(\mathbf{R}^{n}\right)$ invariant under the action of a noncompact semisimple Lie group. For $p=1$ this follows from the work of Greenleaf, Moskowitz and Rothchild [4]. We give a partial solution to the conjecture if $p>1$. Let $G$ be a noncompact connected semisimple Lie group of dimension $n$ and rank $k$. If $G$ has Lie algebra $\mathbb{B} \simeq \mathbf{R}^{n}$ and Killing form $B, m(x)=\phi(B(X, X))$ is an Ad invariant function on $\mathbf{R}^{n}$.

We call $m$ regular on \& if $\phi(t)=o\left(t^{-\alpha}\right)$ for some $\alpha>0$, when $\max (k, n-k) \leqslant 2$; no conditions are imposed if $\max (k, n-k)>2$.

Theorem B. Assume $\phi$ is in $L^{s} \cap L^{\infty}(\mathbf{R})$ for some $s, 0<s<\infty$, and $\phi$ is regular on $\$$. Then $m(X)=\phi(B(X, X))$ is a multiplier of $L^{p}\left(\mathbf{R}^{n}\right)$ if and only if $p=2$.

REMARKS. (a) Let

$$
D=i \frac{\partial}{\partial X_{1}}+\sum_{j=2}^{n} \frac{\partial^{2}}{\partial X_{j}^{2}}+i
$$

Theorem $\mathrm{A}$ and a result of de Leeuw shows $D$ is invertible on $L^{p}\left(\mathbf{R}^{n}\right)$ if and only if $p=2$. E. M. Stein observed that if $P$ is the operator with symbol $\left(y-x^{2}+i\right)$ $\left(y^{2}+x^{2}+i\right), P$ is invertible on some $p \neq 2$, but not all $p, 1<p<+\infty$.

Received by the editors November 16, 1978 .

AMS (MOS) subject classifications (1970). Primary 42A18; Secondary 35E99.

${ }^{1}$ Supported in part by a grant from the NSF. 
(b) The requirement $\phi \in L^{s}$ in Theorems A and B is essential to our method of proof, but examples like $|B(X, X)|^{i t}$ show it is probably too restrictive a condition.

(c) Theorems A and B are adapted to multipliers constant on noncompact quadratic surfaces. The methods of the proof extend to much more general smooth surfaces, a topic we shall discuss elsewhere.

(d) A. Córdoba has observed that a generalization of the techniques of [2] may apply here. This generalization has recently been carried out by Alberto Ruiz.

Sketch of proof. In both proofs, we introduce extra variables, which, when all variables are frozen, make the multipliers behave like multipliers known to be unbounded. In Theorem A we compare $\phi\left(\xi_{1}-\xi_{2}^{2}\right)$ to $\operatorname{sign}\left(\xi_{1}-\xi_{2}^{2}\right)$; in Theorem B we compare $m$ to the characteristic function of the unit ball. We require Kakeya sets to show these latter multipliers unbounded.

PRoposition. Let $N_{k}=2^{k} \log k$. There exists a set $K \subset \mathbf{R}^{2}$ and a collection of $2^{k}$ disjoint rectangles $\left\{R_{j}\right\}$ such that for every $\gamma>0$;

(i) The shorter and longer sides of each $R_{j}$ are bounded above by $N_{k}$, $N_{k}^{2}$ and below by $1 / 4 N_{k}, 1 / 4 N_{k}^{2}$, respectively.

(ii) $|K| \leqslant 20\left(\log \log N_{k}\right)^{-1} \Sigma\left|R_{j}\right|$.

(iii) Let $\bar{v}_{j}=\left(\cos \theta_{j}, \sin \theta_{j}\right)$ denote the direction of the longer side of $R_{\hat{j}}$ Then $\left|\theta_{j}-\pi / 4\right|<\pi / 8$.

(iv) Let $\bar{R}_{j}$ denote $R_{j}+(1+\gamma) \bar{v}_{j}$. Then $\left|\bar{R}_{j} \cap K\right|>1 / 4\left|\bar{R}_{j}\right|$.

The proof is a minor variant of that in [3].

To prove Theorem A, we assume $m$ is a multiplier of $L^{p}$ for $p>2$. We may assume $\phi \geqslant 0, \phi \in L^{1}$, and $\int \phi=1$. By considering the multipliers

$$
m_{t}\left(\xi_{1}, \xi_{2}\right)=m\left(t \xi_{1}, t^{2} \xi_{2}\right)
$$

and following C. Fefferman [3], we obtain an inequality

$$
\left\|\left(\Sigma\left|K_{t}^{j} * f_{j}\right|^{2}\right)^{1 / 2}\right\|_{p} \leqslant C_{p}\left\|\left(\Sigma\left|f_{j}\right|^{2}\right)^{1 / 2}\right\|_{p}
$$

where

$$
K_{t}^{j}(x, y)=\frac{e^{i \bar{\omega}_{j} \cdot \bar{x}_{e} i x^{2} / 4 y}}{t \sqrt{i y}} \hat{\phi}(y / t),
$$

and $C_{p}$ is independent of $t$ and $\left\{\bar{\omega}_{j}\right\}$. Let $f_{j}=\chi_{R_{j}}, t=\gamma \delta N_{k}^{2}$, and

$$
\overline{\omega_{j}}=\left(-1 / 2 \cot \theta_{j}, 1 / 4 \cot ^{2} \theta_{j}\right) \text {. }
$$

We shall show that for $\bar{x} \in \bar{R}_{j}$,

$$
\left|K_{t}^{j} * f_{j}(\bar{x})\right| \geqslant C_{\gamma}
$$


As in [3], it then follows that

$$
C_{p} \geqslant \frac{C_{\gamma}}{40}\left(\log \log N_{k}\right)^{(p-2) / 2 p} \text { for all } k,
$$

a contradiction.

Now,

$$
K_{t}^{j} * f_{j}(\bar{x})=\frac{t^{-1}}{i^{1 / 2}} \int_{R_{j}}\left(x_{2}-y_{2}\right)^{-1 / 2} \exp i \Phi(\bar{x}-\bar{y}) \hat{\phi}\left(\frac{x_{2}-y_{2}}{t}\right) d y,
$$

where

$$
\Phi(\bar{x}-\bar{y})=\frac{\left(x_{2}-y_{2}\right)}{4}\left[\frac{x_{1}-y_{1}}{x_{2}-y_{2}}-\cot \theta_{j}\right]^{2} .
$$

A little geometry shows that when $\bar{x} \in \bar{R}_{j}, \bar{y} \in R_{j}$,

$$
\left|\frac{x_{1}-y_{1}}{x_{2}-y_{2}}-\cot \theta_{j}\right| \leqslant 5 / \gamma N_{k}
$$

and then $|\Phi(\bar{x}-\bar{y})| \leqslant 10 / \gamma$; we write $\exp i \Phi=1+O\left(\gamma^{-1}\right)$ and write $K_{t}^{j} * f_{j}(\bar{x})=M+E$, where

$$
M=\frac{t^{-1}}{i^{1 / 2}} \int_{R_{j}}\left(x_{2}-y_{2}\right)^{-1 / 2} \hat{\phi}\left(\frac{x_{2}-y_{2}}{t}\right) d \bar{y},
$$

and $|E| \leqslant 100 \cdot \gamma^{-5 / 2} \delta^{-1}$. But $\left|x_{2}-y_{2}\right| / t \leqslant 4 / \delta$, so that if $\delta$ is large

$$
\operatorname{Re} \hat{\phi}\left(\frac{x_{2}-y_{2}}{t}\right) \geqslant 1 / 2 \text { and }|M| \geqslant 1 / 4 \gamma^{-3 / 2} \cdot \delta^{-1} \text {. }
$$

If $\gamma>800,|M|>2|E|$, and

$$
\left|K_{t}^{j} * f_{j}(\bar{x})\right| \geqslant \frac{\gamma^{-3 / 2} \delta^{-1}}{8}
$$

This completes the proof.

The proof of Theorem B is similar in spirit, but much more technical, as the convolution kernels cannot be computed explicitly. Using a result of de Leeuw, we restrict $m$ to $\mathbf{R}^{3}$, obtaining $\phi\left(z^{2}-x^{2}-y^{2}\right)$. We then dilate and restrict in $z$, to obtain uniformly bounded radial multipliers $\phi\left(t\left(1-r^{2}\right)\right)$. We show the corresponding convolution kernel $\Phi_{t}(R)$ can be written

$$
\operatorname{Re} \frac{e^{2 \pi i R-\pi i / 4}}{t R^{1 / 2}} \hat{\phi}(R / 2 t)+E(t, R),
$$

where $E(t, R)$ is a finite sum of error terms each of which is $o\left(t^{-\mu} R^{-\nu}\right)$ for some $\mu, \nu, \mu+\nu=3 / 2$, as $t$ and $R$ tend to infinity. With respect to Kakeya 
sets, this is a negligible error, and the main term behaves like the convolution kernel for the disc multiplier. Further details are provided elsewhere.

We wish to thank Professors C. Fefferman and R. Strichartz for many suggestions which were central to our approach. Over the past years we have had extensive conversation with Professor A. Córdoba on these and related Kakeya set matters, and gratefully acknowledge the influence of his work on ours.

\section{BIBLIOGRAPHY}

1. A. P. Calderón, Notes on singular integrals, MIT Press, Cambridge, Mass., 1961.

2. A. Córdoba, The Kakeya maximal function and the spherical summation multipliers, Amer. J. Math. 99 (1977), 1-22.

3. C. Fefferman, The multiplier problem for the ball, Ann. of Math. (2) 94 (1971), 330-336.

4. F. P. Greenleaf, M. Moskowitz, and L. P. Rothchild, Unbounded conjugacy classes in Lie groups and location of central measures, Acta Math. 132 (1974), 225-243.

5. W. Littman, C. McCarthy and N. M. Rivière, The non-existence of $L^{p}$ estimates for certain translation invariant operators, Studia Math. 30 (1968), 219-229.

DEPARTMENT OF MATEHMATICS, PRINCETON UNIVERSITY, PRINCETON NEW JERSEY 08540

DEPARTMENT OF MATHEMATICS, UNIVERSITY OF TEXAS, AUSTIN, TEXAS 78712 